\title{
ESTUDIOS SISTEMÁTICOS, FILOCEN ÉTICOS Y BIOGEOGRÁFICOS EN INSECTOS DE LA REGIÓN AUSTRAL D AMÉRICA DEL SUR
}

\section{C. Domínguez, F. A. Agrain, A. S. Sallenave, E. Ruiz-Manzanos y G. San Blas}

Laboratorio de Entomología, IADIZA CRICYT, CC 507; 5500, Mendoza, Argentina

\section{INTRODUCCIÓN}

La región austral de América del Sur es el área situada por debajo de los $30^{\circ} \mathrm{S}$ (HUMPHRIES \& PARENTI, 1986; CRISCI et al., 1991; ROIG-JUNENTT \& FLORES, 1994) incluyendo las áreas montañosas de los Andes situadas al norte de esta latitud (MORRONE, 1996). Numerosos estudios biogeográficos separan este área del resto del continente americano al considerarla más estrechamente relacionada con biotas de áreas templadas del hemisferio sur, tales como Australia, Nueva Zelanda y África del Sur (CRISCI et al., 1991; ROIG-JUNEENT, 1993, 2000; SANMARTIN \& RONQUIST, 2004). Algunos autores han postulado que América del Sur es un continente de origen híbrido y que debería ser dividido en dos áreas: la templada del sur y la tropical del norte (CRISCI et al., 1991; MORRONE, 2001).

A pesar de los muchos trabajos realizados en el campo de la sistemática y la biogeografía de insectos de América del Sur austral, muchos grupos de insectos aun permanecen relativamente poco conocidos. Existen en la actualidad pocos taxónomos en relación a la enorme diversidad de organismos conocidos y por conocer en esta región.

Los estudios sobre sistemática y biogeografía resultan de gran importancia ya que proveen hipótesis sobre las relaciones de parentesco y la historia biogeográfica del grupo en cuestión, listados actualizados de las especies presentes (catálogos), revisiones taxonómicas de grupos que en muchos casos no han sido revisados en años, actualizaciones de datos de distribuciones de las especies estudiadas, descripciones de nuevas especies y claves para la identificación de los grupos tratados, e información sobre su biología e importancia económica.

La información generada, la obtención de claves y el mejor conocimiento de la distribución de las especies estudiadas, resultan una herramienta fundamental para el desarrollo de trabajos ecológicos y de 
implementación de estrategias de conservación. La adquisición de conocimiento sobre la biodiversidad, y en particular la creación de un inventario de los recursos bióticos, es el punto de partida a la hora de delimitar áreas de conservación. La información básica que proveen estos estudios sistemáticos permite implementar medidas de conservación sobre una base teórica sólida.

En el laboratorio de Entomología del Instituto Argentino de Investigaciones de las Zonas Áridas (IADIZA), ubicado en el Centro Regional de Investigaciones Científicas y Tecnológicas (CRICYT), en Mendoza, Argentina, se están realizando revisiones taxonómicas, análisis filogenéticos y biogeográficos de distintos grupos de insectos, con particular énfasis en los representantes australes de América del Sur. Principalmente se están realizando estudios sobre las familias de Coleópteros Carabidae, Curculionidae y Tenebrionidae, además de estudios ecológicos sobre insectos acuáticos, hormigas y arácnidos. El estudio de estas tres familias de Coleópteros tiene una larga trayectoria en el laboratorio de Entomología, con numerosas contribuciones de los investigadores Sergio A. Roig-Juñent, Adriana E. Marvaldi y Gustavo E. Flores. Han sido tratados distintos aspectos de estos grupos tales como aspectos biogeográficos, filogenéticos (tanto moleculares como morfológicos) y taxonómicos; además, se han descrito gran cantidad de taxa nuevos en los últimos 15 años, fundamentalmente para Argentina y Chile. Estos estudios pueden consultarse en www.cricyt.edu.ar/entomologia.

El objetivo de este trabajo es mostrar las nuevas líneas de trabajo que han surgido en nuestro laboratorio durante los últimos años sobre los siguientes taxa: Fanniidae (Diptera), Megalostomina (Coleoptera: Chrysomelidae); Bembidiini (Coleoptera: $\mathrm{Ca}$ rabidae); Noctuidae (Lepidoptera); Melolonthinae (Coleoptera: Scarabaeidae).

\section{DIVERSIDAD Y ESTADO DEL CONOCI- MIENTO DE LA SUBTRIBU MEGALOSTO- MINA (COLEOPTERA: CHRYSOMELIDAE) EN AMÉRICA DEL SUR}

La subtribu Megalostomina (5 géneros, 141 especies) (MOLDENKE, 1970) es un interesante grupo de Crisomélidos (Subfamilia: Cryptocepha- linae) debido a su peculiar morfología, biología y adaptación a zonas áridas y semiáridas. Se distribuye en América tropical desde el sudoeste de EE.UU. hasta Argentina. Megalostomina está ricamente representada en las regiones xéricas de América del sur y en Argentina se encuentran algunos endemismos, en particular en la región del monte xerófilo. Los datos de distribución, así como los de asociación con plantas huéspedes están siendo actualizados para determinar claramente el número de endemismos y la importancia ecológica de su asociación con plantas nativas.

El conocimiento actual de la sistemática de Megalostomina es muy fragmentario, pues las revisiones existentes son parciales, considerando sólo las especies distribuidas en determinados países. Por otra parte, muchas de las descripciones originales resultan ambiguas al considerar caracteres con gran variación intraespecífica, como por ejemplo, los patrones de coloración de los élitros. Los ejemplares tipo de varias especies no son fácilmente accesibles, o bien se han perdido o dañado. Si bien en la literatura existen algunas propuestas sobre las posibles relaciones filogenéticas entre las especies, éstas son contradictorias, como por ejemplo MONRÓS (1953) y MOLDENKE (1981), y éstas deben ser contrastadas mediante una metodología formal. Del mismo modo, la monofilia del género y sus subgéneros no ha sido verificada con estudios filogenéticos.

Una peculiaridad de las especies de este grupo de coleópteros es su compleja y poco conocida biología, fuera de lo común en comparación con la de la mayoría de los crisomélidos, ya que en estado larval presentan mirmecofilia o submirmecofilia, alimentándose dentro o cerca de los hormigueros, de huevos, exuvias, excretas y cuerpos de hormigas, en tanto que el adulto es estrictamente fitófago, alimentándose principalmente de hojas de leguminosas (Fabaceae) (JOLIVET \& HAWKESWOOD, 1995). Esta asociación, con hormigas en estado larval, y con plantas en estado adulto, plantea interesantes aspectos coevolutivos, nunca antes evaluados en un contexto filogenético.

La revisión y delimitación de los grupos naturales y las especies de Megalostomina constituirá un avance en el conocimiento de uno de los taxa más representativos de las regiones áridas de América 
del Sur. La elucidación de la filogenia de este grupo permitirá analizar los procesos coevolutivos que involucran a estos coleópteros y sus huéspedes, y servirá de base para realizar un análisis biogeográfico histórico. Actualmente se está trabajando en la revisión del género Megalostomis Chevrolat, uno de los más representativos y diversos de la subtribu con 53 especies y 20 subespecies, habiéndose ya terminado la revisión del subgénero Megalostomis (Scaphigenia) Lacordaire. Este proyecto incluye la proposición de una filogenia robusta de la subtribu Megalostomina, que permita interpretar las relaciones entre sus géneros y subgéneros, así como su posición respecto al resto de la tribu Clytrini Lacordaire y la subfamilia Cryptocephalinae Gyllenhal. También se incluirá la revisión de Megalostomis (Figura 1) y los géneros más representativos de la región, y un análisis biogeográfico de la subtribu.

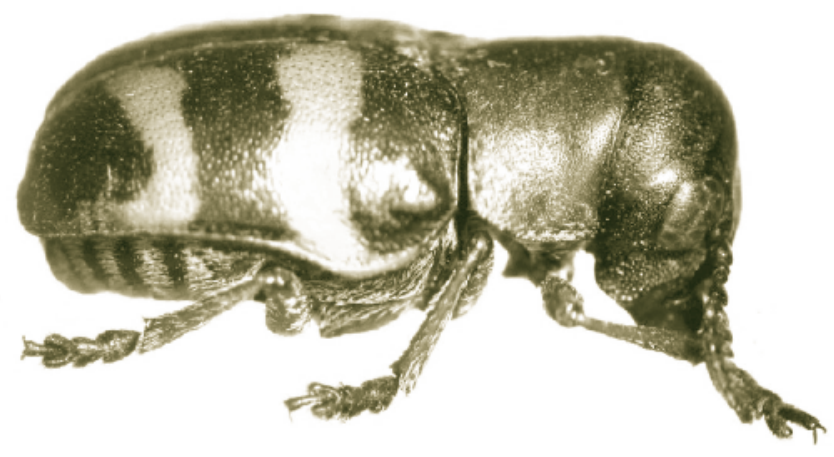

Figura 1. Megalostomis (Scaphigenia) gazella Lacordaire.

\section{DIVERSIDAD Y ESTADO DEL CONOCI- MIENTO DE LA TRIBU BEMBIDIINI (CO- LEOPTERA: CARABIDAE), EN AMERICA DEL SUR AUSTRAL}

En la Argentina se conocen unas 700 especies de carábidos pertenecientes a 163 géneros, de los cuales casi el $40 \%$ son endémicas de este país. La tribu Bembidiini posee su mayor diversidad en el sur de Sudamérica (Argentina, Chile y Uruguay) donde está representada por 248 especies pertenecientes a 31 géneros. La última revisión de la tribu fue realizada por JEANNEL (1962) y los trabajos posteriores sólo han sido contribuciones parciales. Dentro de Bembidiini, la subtribu Bembidiina (Figura 2) es la que presenta mayor diversidad con gran cantidad de géneros y especies, la mayoría endémicos de la región, siendo todas sus especies endémicas de América del Sur austral. Al igual que en el caso anterior, ningún trabajo posterior al de JEANNEL (1962) ha realizado un estudio completo, ni ha planteado las relaciones de los grupos de especies de América del Sur con las restantes especies de Bembidiina en el mundo. Si bien el trabajo de JEANNEL (1962) ha sido de gran importancia para la entomofauna de la región austral [al igual que los trabajos de SOLIER (1849) y GERMAIN (1906)], es necesaria la revisión y continuación de estos estudios en la tribu Bembidiini, así como en studios filogenéticos y biogeográficos más completos. Además, en las últimas décadas, han

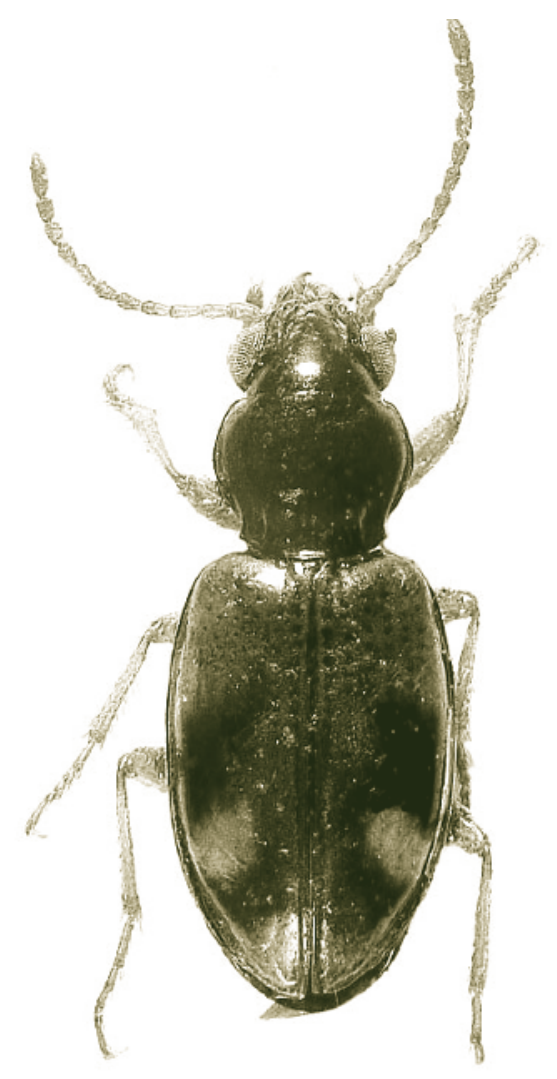

Figura 2. Bembidion (Nothonepha) lonae JensenHaarup. 
aumentado notablemente las colectas de material de este grupo, reconociéndose gran cantidad de nuevos taxa que deberían ser descritos y analizados (ROIGJUNEENT \& DOMÍNGUEZ, 2001).

Los Bembidiina cumplen un importante rol ecológico ya que son depredadores de otros insectos, lo que los convierte en uno de los principales grupos controladores de la fauna del suelo. La revisión y delimitación de los grupos naturales y sus especies en la subtribu Bembidiina constituirá un avance en el conocimiento de uno de los taxa más representativos del cono sur de América del Sur. La importancia de este estudio no sólo comprende aspectos sistemáticos, sino también biogeográficos ya que se ampliará el conocimiento de la distribución geográfica de los representantes de esta tribu.

ESTUDIOS SOBRE LA SUBFAMILIA MELOLONTHINAE (COLEOPTERA: SCARABAEOIDEA: SCARABAEIDAE) EN AMÉRICA DEL SUR AUSTRAL

Melolonthinae MacLeay (Figura 3) es una de las subfamilias más diversas de Scarabaeidae en el mundo y es la más diversa de Scarabaeidae en Ar-

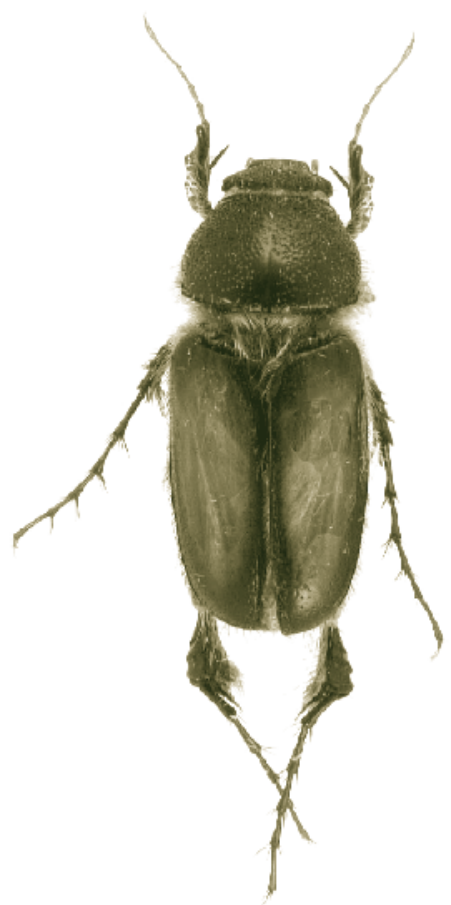

Figura 3. Anahi guaraniticus Martínez. gentina. En el mundo, la subfamilia Melolonthinae, incluye más de 11.000 especies y 750 géneros agrupados en 11 tribus, y en la Argentina, se encuentran representadas seis de estas tribus y 33 géneros. La monofilia del grupo es incierta y no hay consenso entre los diferentes autores sobre su composición ni sobre la posición sistemática de muchos de los taxa que la componen. La clasificación a nivel de tribu es confusa debido a la falta de definición de cada grupo y de la falta de consistencia en el uso de caracteres diagnósticos. Pocos géneros de Melolonthinae de Argentina han sido revisados y la mayoría de los taxa descritos han sido publicados antes de 1940. Muchas especies nuevas se han descubierto en los últimos 50 años pero no existen revisiones modernas que esclarezcan la situación sistemática y composición de cada uno de los géneros. La mayoría de las descripciones genéricas y específicas son pobres e inadecuadas, como consecuencia, se han generado un alto número de sinonimias, así como géneros y especies con dudosa ubicación sistemática. Esta situación provoca que las determinaciones resulten difíciles, si no imposibles, sin el estudio de material tipo, problema agravado por la falta de claves para la identificación de la mayoría de las especies.

Desde el punto de vista biogeográfico, la fauna de Melolonthinae de Argentina puede dividirse en dos grupos principales, el Neotropical y el Patagónico. El alto grado de endemicidad de grupos de Melolonthinae en el país hace que la fauna Argentina sea de particular importancia en estudios de conservación, diversidad, biogeografía y evolución. En Argentina hay especies que constituyen importantes plagas de cultivos como por ejemplo, el género Macrodactylus Dejean, una seria plaga para rosales y frutales, y el género Demodema Blanchard, plaga del girasol (DI IORIO, 2004). Los daños a los cultivos son causados por la larva de los escarabajos, que generalmente se alimenta de las raíces. Estudios en la sistemática y filogenia de Melolonthinae aplicando técnicas modernas supondrían, un enorme avance, tanto para el conocimiento aplicado en el control de las especies plaga, como una importante base para subsiguientes estudios ecológicos y biogeográficos.

Actualmente se está realizando la revisión de los géneros neotropicales de una de las tribus de Melolonthinae presentes en Argentina, Pachydemini 
Reitter, 1902, incluyendo estudios sobre la filogenia y biogeografía de esta tribu. Durante los dos últimos ańos se han realizado diferentes colectas y revisiones de colecciones generando la descripción de al menos 2 géneros y 16 especies nuevas en el Neotrópico, la mayoría de ellas de Argentina (OCAMPO \& SMITH, 2006, OCAMPO, en prep.; OCAMPO \& RUIZ-MANZANOS 2007a, en prensa; RUIZMANZANOS \& OCAMPO, en prep.). Además, se están realizando diversos trabajos sobre la subfamilia Melolonthinae (OCAMPO \& RUIZ-MANZANOS 2007b, en prensa).

\section{DIVERSIDAD Y ESTADO DEL CONOCI- MIENTO DEL GÉNERO AGROTIS (LEPI- DOPTERA: NOCTUIDAE) EN AMERICA DEL SUR AUSTRAL}

La subfamilia Noctuinae está constituida por 52 géneros de los cuales 39 están representados en Sudamérica. Entre los géneros de Noctuinae uno de los más diversos y complejos taxonómicamente es el género Agrotis Ochsenheimer (1816) (Figura 4). Las especies del género Agrotis muestran una distribución mundial y están asociadas a plantas monocotiledóneas. Actualmente se reconocen 320 especies de Agrotis a nivel mundial, de las cuales 88 están representadas en Sudamérica (POOLE, 1989). A pesar de ser un género tan rico en especies, ha sido poco estudiado y solamente hay disponible una revisión para las especies norteamericanas de la tribu Agrotini Rambur realizada por LAFONTAINE (2004). Para Sudamérica no se han realizado revisiones del género, la única información disponible son las publicaciones originales y varias revisiones parciales realizadas por Köhler para las especies argentinas en las décadas del 40 y 60 . Esto pone en evidencia el bajo nivel de estudio en el que se encuentra el género y la necesidad de una revisión que incluya todas las especies sudamericanas utilizando metodologías modernas

La tribu Agrotini está conformada por géneros morfológicamente muy similares, lo que ha provocado confusiones respecto a la caracterización de los mismos y a las especies que cada uno incluye. Para la caracterización de los distintos géneros se han utilizado caracteres morfológicos útiles para otros

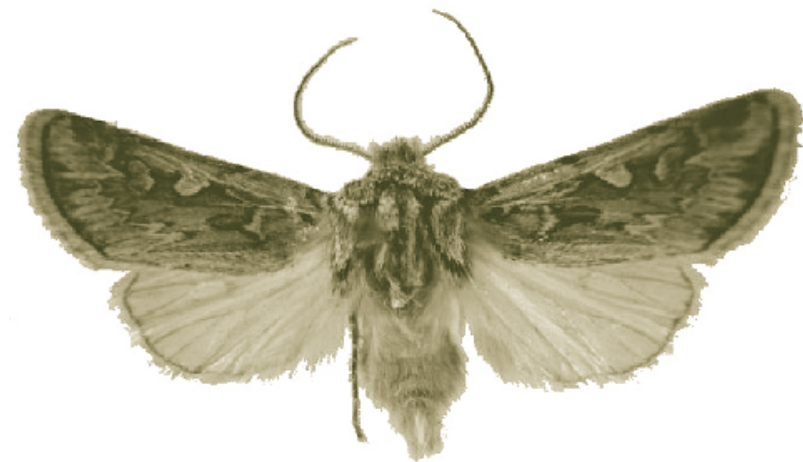

Figura 4. Agrotis gentilii Köhler.

grupos, pero que han demostrado no ser útiles para este grupo, como caracteres diagnósticos a nivel genérico (por ejemplo, forma de las antenas, longitud de las pectinas, forma de la frente). Esto mismo ha ocurrido con el género Agrotis, que también incluye especies muy similares entre sí, creando un gran número de sinonimias. Para dilucidar estos problemas es necesario realizar estudios de genitalia masculina y femenina, al igual que estudios más profundos de la morfología externa, para poder así encontrar nuevos caracteres que definan los géneros, los grupos dentro de los mismos y que caractericen mejor las especies.

Como parte de un trabajo monográfico sobre las especies de Agrotis se han recopilado las descripciones originales de cada una de las especies, y la bibliografía más relevante sobre el género y se han hecho disecciones de las genitalias y cuerpo entero de cada una de las especies Argentinas. En un futuro se planea incluir un mayor número de taxa de diversos géneros sudamericanos, se incluirán datos morfológicos de los nuevos taxa y datos moleculares.

La revisión y análisis filogenético del género revelarán si éste constituye un grupo natural y las relaciones entre sus especies. A su vez, aportará nuevos caracteres diagnósticos a nivel genérico y específico, ayudando a la identificación de los mismos. Dicha información servirá para conformar la primera clave para identificar las especies sudamericanas del género. Junto con la revisión realizada por LAFONTAINE (2004), este trabajo completaría el estudio del género para el continente americano. 
DIVERSIDAD Y ESTADO DEL CONOCIMIENTO DE LA FAMILIA FANNIIDAE (DIPTERA: CALYPTRATAE) EN AMÉRICA DEL SUR AUSTRAL

La familia Fanniidae (Diptera: Calyptratae, Muscoidea) contiene unas 300 especies descritas; su distribución comprende casi todas las regiones faunísticas, aunque la mayor diversidad se halla principalmente en las zonas templadas de ambos hemisferios (CHILLCOTT, 1961; HENNIG, 1965; ROZKOSNY et al., 1997). Esta amplia distribución de las especies es explicada por los hábitos alimentarios de las larvas, en su mayoría detritívoras, y por su gran capacidad de dispersión (ROZKOSNY et al., 1997). La mayor cantidad de especies descritas corresponde a la región Holártica (unas 260 especies). En la región Neotropical se conocen sólo unas 75 especies asignadas a los géneros Fannia y Euryomma (CARVALHO et al., 2003) y en Argentina y Chile se conocen 25 especies del género Fannia (DOMÍNGUEZ, 2005) y cinco del género Euryomma. De éstas, 14 son endémicas de Argentina y Chile y el grupo "anthracina", compuesto de seis especies es endémico de la Patagonia.

De las especies de Fannia citadas para la Argentina, se destacan por su mayor importancia desde el punto de vista sanitario las siguientes: $F$. albitarsis

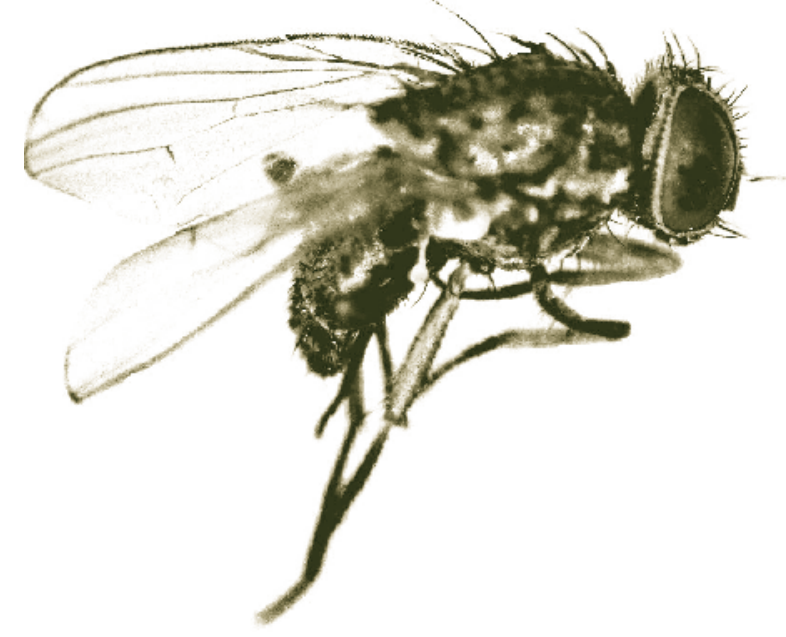

Figura 5. Fannia fusconotata Rondani.
Stein, F. canicularis L., F. femoralis Stein, F. fusconotata Rondani (Figura 5) y F. scalaris Fabricius. Sus larvas son saprófagas y se desarrollan en un amplio rango de sustratos. Estas especies puede portar ciertas bacterias como Staphyloccocus aureus y Escherichia coli (ROZKOSNY et al., 1997) y son capaces de transferir protozoos parásitos, algunas especies de Taenia y nemátodos (GREENBERG \& GEORGE, 1985) y ocasionan problemas en plantas de procesamiento de alimentos. En Mendoza, Argentina, la especie $F$ albitarsis ha sido reconocida como una plaga de granjas avícolas (DOMÍNGUEZ, datos no publicados). Además, las larvas de F. canicularis y F. scalaris, así como de F. fusconotata (citada solamente para Argentina) han sido reconocidas como causantes accidentales de miasis en el hombre y en el ganado (MAZZA \& ORIBE, 1939; JAMES, 1947; CHILLCOTT, 1961; OLIVA, 1997; ROZKOSNY et al., 1997). Dos especies, F canicularis y F albitarsis han sido encontradas en gran cantidad en un estudio realizado en una granja del sur de la provincia de Buenos Aires en Argentina (PEROTTI, 1998).

No existen trabajos que mencionen la importancia sanitaria de las especies del género Euryomma posiblemente debido al desconocimiento que se tiene del mismo, no existiendo descripciones de los estados inmaduros, salvo para la especie cosmopolita E. peregruinum (HOLLOWAY, 1985). Las especies de E. campineira y E. carioca han sido citadas como moscas sinantrópicas (LINHARES, 1981; ALMEIDA et al., 1985). No se tienen datos acerca de su incidencia sanitaria.

En el primer estudio filogenético de las especies de la familia Fanniidae, que estamos realizando se demuestra que el género Fannia constituye un grupo monofilético, y que las especies neotropicales muestran mayor afinidad con grupos de especies neocelandeses y australianos, que con representantes holárticos, como habían sugerido CHILLCOTT (1961) y HENNIG (1965). En el estudio de la historia biogeográfica de este grupo, encontramos que las especies australes de Fanniidae muestran un patrón de distribución muy congruente con la separación de Gondwana, lo cual indica una historia biogeográfica distinta a la planteada por CHILLCOTT (1961) y HENNIG (1965), la cual tenía un enfoque holarticista. Un mejor conocimiento biogeográfico 
permitirá además utilizar este grupo de organismos en estudios de definición de áreas de endemismos, e importancia sanitaria.

El conocimiento sobre la morfología de los estados inmaduros de las especies de Fannia presentes en América del sur es muy escaso, mientras que las larvas de las especies Neárticas, europeas y neocelandesas han sido descritas por CHILLCOTT (1961) y HOLLOWAY (1985).

El conocimiento de la morfología de los estados inmaduros de especies de Fanniidae es necesario para fines aplicados (relacionados con la importancia sanitaria de las especies) y sistemático-evolutivos. Así, la información sobre los estados inmaduros de las especies consideradas perjudiciales tendrá aplicación en estrategias de control de plagas y aspectos relacionados con la entomología forense. Asimismo, el estudio de los estados larvales permitirá disponer de una nueva fuente de caracteres para el análisis filogenético de Fannia y Euryomma y conocer mejor los patrones evolutivos del grupo.

Muy pocos trabajos tratan sobre la cría y la duración de los ciclos de vida de las especies de Fannia de importancia sanitaria. Para la mosca doméstica menor, Fannia canicularis, pueden citarse los trabajos de MANDEVILLE et al. (1988), MULLENS et al. (2002) y GREENBERG \& GEORGE (1985). La única especie de Fannia cuyo ciclo biológico ha sido estudiado exhaustivamente es Fannia pusio (LINHARES, 1978, 1981; COURI, 1992a, 1992b).

\section{RESULTADOS PRELIMINARES}

Para los cinco grupos sistemáticos mencionados se han revisado la mayor parte de las colecciones nacionales y muchas colecciones extranjeras así como el material tipo de cada especie. Se han realizado numerosas colectas en distintos ambientes de Argentina. Existen, sin embargo, varias regiones del país que han sido pobremente exploradas y donde, muy posiblemente, existan especies aun desconocidas para la ciencia, en particular de los grupos de insectos mencionados en este trabajo.

Para cada grupo se han realizado o se están realizando colecciones de referencia, que permiten la correcta identificación del material. La información, tanto bibliográfica como la extraída de los materiales examinados en los distintos museos, han permitido la elaboración de bases de datos para cada una de las especies estudiadas, en donde se incluyen datos sobre la historia taxonómica y citas bibliográficas. Estas bases de datos facilitarán el trabajo de identificación de materiales y sobre todo la detección de problemas taxonómicos que puedan estar presentes, tales como sinonimias. Asimismo, la revisión de material proveniente de colecciones y de nuevas colectas permite la actualización y ampliación del conocimiento de la distribución geográfica de las especies de cada grupo. Se está recopilando una base de datos georeferenciada de las distribuciones de cada especie. Esta información será utilizada para la elaboración de mapas, inferencia de datos climáticos relacionados con la distribución de las especies, estudios de biogeografía histórica, análisis de endemicidad, etc.

En cada grupo se ha comenzado con la redescripción de las especies ya conocidas y la elaboración de claves para la identificación. En la mayoría de los casos, éstas están basadas en caracteres utilizados tradicionalmente en cada uno de los grupos, pero a ellos se han sumado caracteres nuevos como caracteres de la genitalia tanto masculina como femenina.

Hasta el momento, se han descrito un género y ocho especies de Pachydemini (Melolonthinae) (OCAMPO \& SMITH 2006, OCAMPO, en prep.; OCAMPO \& RUIZ-MANZANOS 2007a, en prensa; RUIZ-MANZANOS \& OCAMPO, en prep.), un género y especie nueva de Bembidiini, Argentinatachoides (SALLENAVE et al., en prensa) y tres nuevas de Fannia (Fanniidae).

En cuanto al análisis Cladístico, se ha comenzado con la elaboración de las matrices de datos morfológicos. La filogenia obtenida se utilizará para inferir patrones de biogeografía histórica.

\section{CONCLUSIONES}

Para la mayoría de los grupos de insectos no existen revisiones, o las que se encuentran fueron hechas a principio del siglo pasado y por lo tanto requieren ser actualizadas, tratándose en la mayor parte de los casos de revisiones parciales.

Además, las hipótesis de relaciones de parentesco existentes en general no han sido planteadas en un 
marco filogenético y esto ha llevado a un sistema de clasificación artificial en muchos grupos. Por todo ello, son necesarios nuevos estudios filogenéticos con la aplicación de métodos modernos que proveerán clasificaciones naturales que podrían ser de utilidad en otros campos tales como la conservación, ecología y biogeografía.

Estudios recientes han demostrado que la fauna entomológica, en particular de los grupos aquí mencionados, ha sido subestimada. El alto número de taxones recientemente descritos o en proceso de ser descritos es una prueba de ello. El estudio de la fauna de América del Sur Austral puede brindar mayor soporte a las hipótesis biogeográficas y sistemáticas actuales de estos grupos, o bien ofrecernos nuevas e interesantes hipótesis al respecto.

Como mencionamos anteriormente, la información básica generada por los estudios taxonómicos, las hipótesis filogenéticas, y los estudios biogeográficos resultan fundamentales para el desarrollo de trabajos ecológicos y de implementación de estrategias de conservación. Estos estudios básicos responden a preguntas tales como: qué especies hay, dónde viven y cómo se relacionan entre sí (desde el punto de vista filogenético), llevando de esta manera a la identificación de endemismos, grupos relictos, asociaciones planta-insecto, etc., parámetros que permiten tomar decisiones en cuanto a áreas prioritarias de conservación.

\section{AGRADECIMIENTOS}

Agradecemos a Federico Ocampo su invitación a participar en este proyecto y junto a Sergio RoigJuñent, por la lectura crítica del manuscrito. Este estudio ha sido financiado por CONICET, PICT 01-11-120 Biodiversidad de Artrópodos de Alta Montańa (FONCyT).

\section{BIBLIOGRAFIAA CITADA}

AGRAIN, F. A, S. A. ROIG-JUNEENT \& M. C. DOMÍNGUEZ. 2007. Taxonomic revision and preliminary cladistic analysis of the subgenus Megalostomis (Scaphigenia) (Coleoptera: Chrysomelidae: Cryptocephalinae). Insect Syst. Evol. (en prensa)
CARVALHO, C. J. B. DE, A. C. PONT, M. S. COURI, \& D. M. PAMPLONA. 2003. Catalogue of the Fanniidae (Diptera) of the Neotropical Region. Zootaxa 219: 1-32.

CHILLCOTT, J. G. 1961. A revision of the Neartic species of Fanniinae (Diptera: Muscidae). Can. Entomol. 1-295.

COURI, M. S. 1992a. Oogenese em Fannia pusio (wiedemann, 1830) e Fannia heydenii (Wiedemann, 1830) (Diptera, Fanniidae). Rev. Bras. Zool. 7 (1991): 59-67.

COURI, M. S. 1992b. Influencia da dieta no desenvolvimiento oogenico de Fannia pusio (Wiedemann,1830) (Diptera, Fanniidae). Rev. Bras. Zool. 7 (1991): 85-88.

CRISCI, J. V., M. CIGLIANO, J. J. MORRONE \& S. A. ROIG-JUNEENT. 1991. Historical Biogeography of Southern South America. Syst. Zool. 40: 152-171.

DI IORIO, O. R. 2004. Coleoptera: Scarabaeidae: Melolonthinae. En: H. A. Cordo, G. Logarzo, K. Braun \& O. R. Di Iorio (directores). Catalogo de Insectos Fitófagos de la Argentina y sus plantas asociadas. Sociedad Entomológica Argentina ediciones. Buenos Aires. 734 pp.

DOMÍNGUEZ, M. C. 2005. Revisión sistemática y análisis Cladístico de las especies de Fannia (Robineau Desvoidy) de América del Sur austral. Tesis. Universidad Nacional de Córdoba.

ERBER, D. 1988. Biology of Camptosomata Clytrinae - Cryptocephalinae - Chlamisinae - Lamprosomatinae. En: P. Jolivet, E. Petitpierre \& T. H. Hsiao (eds.), Biology of Chrysomelidae vol. III. Kluwer Academic Publishers, The Netherlands. Pp. 513-552.

GERMAIN, P. 1906. Apuntes entomológicos: Bembidios chilenos. Anales de la Universidad. Santiago de Chile.

GREENBERG, B. \& J. George. 1985. Fannia canicularis. En: P. Singh \& R. F. Moore (eds.), Handbook of Insect Rearing. Elsevier, Amsterdam, Oxford etc. 2. Pp: 93-96.

HENNIG, W. 1965. Vorarbeiten zu einem phylogenetische.n system der Muscidae (Diptera: Cyclorrhapha). Stutt. Beitr. Naturk. 141: 1-100.

HOLLOWAY, B. A. 1985. Larvae of New Zealand Fanniidae (Diptera: Calyptrata). New Zeal. J. Zool. 11: 239-257. 
HUMPHRIES, C. J. \& L. R. PARENTI. 1986. Cladistic biogeography. Oxford, Clarendon press.

JAMES, M. T. 1947. The flies that cause myiasis in man. United States Department of Agriculture Miscellaneous Publications 631: 175pp.

JEANNEL, R. 1962. Les trechides de la paleantartique occidentale. En: C. D. Delamare Debouteville, \& E. Rapoport, (eds.), Biol. Amer. Austr. 1, CHRS et CNIT, Paris. Pp. 527-655.

JOLIVET, P. \& HAWKESWOOD, T. J. 1995. Host-plants of Chrysomelidae of the world. Backhuys, Leiden, 281pp.

LAFONTAINE, J. D. 2004. Noctuoidea, Noctuidae (part): Noctuinae, Agrotini. En: R.W. Hodges, D.R. Davis, D.C. Ferguson, E.G. Munroe, \& J.A. Powell, (eds.) The Moths of America North of Mexico, fasc. 25.1, Allen Press, Lawrence, Kansas. 385 pp.

LINHARES, A. X. 1978. Estudos sobre eclosao em ovos de Fannia pusio Wiedemann (Diptera; Muscidae) em laboratorio. Cienc. Cult. 30: 603.

LINHARES, A. X. 1981. Synantropy of Muscidae, Fanniidae and Anthomyiidae (Diptera) in the city of Campinas, Sao Paulo, Brasil. Rev. Bras. Entomol. 25: 231-243.

MANDEVILLE J. D., B. A. MULLENS \& J. A. MEYER. 1988. Rearing and host age suitability of Fannia canicularis (L.) (Diptera: Muscidae) for parasitization by Muscidifurax zaraptor Kogan and Legner (Hymenoptera: Pteromalidae). Can. Entomol. 120: 153-159.

MAZZA, S. \& H. R. ORIBE. 1939. Miasis urinaria por Fannia fusconata Rondani, en Formosa. Investigaciones sobre Dipteros Argentinos. I Miasis. Jujuy, Publicaciones Misión de Estudios de Patología Regional Argentina: 66-69.

MOLDENKE, A. R. 1970. A revision of the Clytrinae of North America, North of the itsmus of Panama (Col: Chrys). Department of Biological Sciences, Stanford, University California.

MOLDENKE, A. R. 1981. A generic reclassification of the New World Clytrinae. With descriptions of new species. Ent. Arb. Mus. Frey 29: 75-116.

MONRÓS, F. 1953. Revisión sistemática de las especies de Clytrinae de la Argentina, Paraguay, Uruguay y Chile (Col., Chysomelidae). Acta Zool. Lilloana 14: 5-274.
MORRONE, J. J. 2001. Biogeografía de América Latina y el Caribe. M y T- Manuales \& Tesis SEA, vol.3. Zaragoza. $148 \mathrm{pp}$.

MORRONE, J. J. 1996. The biogeographical Andean subregion: A proposal exemplified by arthropod taxa (Arachnida, Crustacea, and Hexapoda). Neotrópica 42: 103-114.

MULLENS B. A., C. E. SZIJI \& N. C. HINKLE. 2002. Oviposition and Development of Fannia spp. (Diptera: Muscidae) on Poultry Manure of Low Moisture Levels. Physiological and Chemical Ecology. 31: 588-593.

OLIVA, A.1997. Insectos de interés forense de Buenos Aires (Argentina). Primera lista ilustrada y datos bionomicos. Rev. Mus. Argent. Cienc. Nat. "Bernardino Rivadavia" Inst. Nac. Inv. Cien. Nat. VII: $13-59$.

PEROTTI, A. 1998. Mosca sinantrópicas (Diptera: Muscidae y Fanniidae) asociadas a producciones avícolas del centro-sudeste bonaerense. Nat. Neotrop. 29: 145-154.

OCAMPO, F. C. \& RUIZ-MANZANOS, E. 2007a. A Revision of the Neotropical Genus Anahi Martínez (Coleoptera: Scarabaeidae: Melolonthinae) with the Description of Two New Species. Neotr. Entomol. (en prensa).

OCAMPO, F. C. \& RUIZ-MANZANOS, E. 2007b. Scarabaeidae. En: G. O. Debandi, L. E. Claps \& S. A. Roig-Juñent (eds.), Biodiversidad de artrópodos argentinos, vol. 2. (en prensa).

OCAMPO, F. C. \& SMITH, A. B. T. 2006. Puelchesia gracilis, a new genus and species of Pachydemini endemic to the Monte in Argentina (Coleoptera: Scarabaeidae: Melolonthinae). Zootaxa 1349: 53-62.

OCHSENHEIMER, F. 1816. Die Schmetterlinge von Europa. Leipzig: Bey G. Fleischer dem Jüngern Vol. 4, 66.

POOLE, R. W. 1989. Lepidopterorum Catalogus (N.S.) Noctuidae. Part. 1-2. E.J. Brill Flora \& Fauna Publications, 118: 1013 pp.

REITTER, E. 1902. Bestimmungs-Tabelle Melolonthidae aus der europäischen Fauna und den angrenzenden Ländern, enthaltend die Gruppen der Pachydemini, Sericini und Melolonthini. Verhand. d. Naturf. V. Brünn, 40, 1901 (publicado en 1902): 93-136. 
ROIG-JUNENET, S. A. 1993. Cnemalobini, una tribu de Carabidae (Coleoptera) endémica de América del Sur. Acta Entomol. Chilena 16: 37-67.

ROIG-JUNENT, S. A. 2000. The subtribes and genera of the tribe Broscini (Coleoptera: Carabidae): Cladistic analysis, taxonomic treatment, and biogeographical considerations. Bull. Amer. Mus. Nat. Hist. 255: 1-90.

ROIG-JUNEENT, S. A., \& DOMÍNGUEZ, M. C. 2001. Diversidad de la familia Carabidae (Coleoptera) en Chile. Rev. Chil. Hist. Nat. 74: 549-571. 2001.

ROIG JUÑENT, S. A., \& G. E. FLORES. 2001. Historia biogeográfica de las áreas áridas de América del Sur austral. En: J. J. Morrone \& J. Llorente Bousquets (eds.), Introducción a la biogeografía en Latinoamérica: Teorías, conceptos, métodos y aplicaciones. Las Prensas de Ciencias,
Facultad de Ciencias, UNAM, México, D. F. Pp. 257-266.

ROZKOSNY, R., G. FRANTISEK \& A. C. PONT. 1997. The European Fanniidae (Diptera). Acta Sci. Nat. Brno. 31: 1-80.

SALLENAVE, S., T. L. ERWIN, \& S. ROIGJUNENT. 2007 Argentinatachoides, a new genus from Argentina putatively related to the Australian-Tasmanian Tasmanitachoides Erwin 1972 (Coleoptera: Carabidae: Bembidiini). Ann. Carnegie Mus. (en prensa).

SANMARTÍN, I., \& F. RONQUIST. 2004. Southern Hemisphere Biogeography Inferred by event-Based models: Plant versus Animal patterns. Syst. Biol. 53: 216-243.

SOLIER, A. J. J. 1849. Coleópteros. En: C. Gay, (Ed.), Historia Física y Política de Chile. Zoología volumen 4. Pp. 105-272. 